\title{
Homologous multi-points warping: an algorithm for automatic 3D facial landmark
}

\begin{abstract}
Over the decade scientists have been researching to know whether face recognition is performed holistically or with local feature analysis which has led to the proposition of various advanced methods in face recognition, especially using facial landmark. The current facial landmark methods in 3D are mathematically complex, contain insufficient landmarks, lack homology and full of localization error due to manual annotation. This paper proposes an Automatic Homologous Multi-Points Warping (AHMW) for 3D facial landmarking, experimented on three datasets using 500 landmarks (16 anatomical fixed points and 484 sliding semi-landmarks) by building a template mesh as a reference object and thereby applies the template to each of the targets on three datasets. The results show that the method is robust with minimum localization error (Stirling/ESRC:0.077; Bosphorus:0.088; and FRGC v2: 0.083).
\end{abstract}

Keyword: 3D facial landmark; Landmark algorithm; Homologous facial points; 3D morphology; TPS warping; Localization error Introduction 